\title{
Cryspovirus: a new genus of protozoan viruses in the family Partitiviridae
}

\author{
Max L. Nibert • Keith M. Woods • Steve J. Upton • \\ Said A. Ghabrial
}

Received: 29 June 2009/Accepted: 3 September 2009/Published online: 25 October 2009

(C) Springer-Verlag 2009

\begin{abstract}
The family Partitiviridae includes plant and fungal viruses with bisegmented dsRNA genomes and isometric virions in which the two genome segments are packaged separately and used as templates for semiconservative transcription by the viral polymerase. A new genus, Cryspovirus, has been approved for this family. Its name is based on that of the host genus, Cryptosporidium, which encompasses several species of apicomplexan parasites that infect a wide range of mammals, birds, and reptiles, and are a major cause of human diarrheal illness worldwide. The type species of the new genus is Cryptosporidium parvum virus 1 . Distinguishing characteristics include infection of a protozoan host, a smaller capsid protein than found in other members of the family Partitiviridae, and sequence-based phylogenetic divergence.
\end{abstract}

\section{Introduction}

The family Partitiviridae encompasses a wide range of viruses characterized by isometric virions $\sim 35 \mathrm{~nm}$ in diameter and a bisegmented dsRNA genome coding for

\section{L. Nibert ( $\square)$}

Department of Microbiology and Molecular Genetics, Harvard Medical School, 200 Longwood Avenue, Boston, MA 02115, USA

e-mail: mnibert@hms.harvard.edu

K. M. Woods · S. J. Upton

Division of Biology,

Kansas State University,

Manhattan, KS 66506, USA

\section{S. A. Ghabrial}

Department of Plant Pathology,

University of Kentucky,

1405 Veterans Drive, Lexington, KY 40546, USA only an RNA-dependent RNA polymerase (RdRp) on one segment and a capsid protein (CP) on the other $[8,9]$. The two genome segments are packaged in separate virions [3] and used as templates for semiconservative transcription by the virion-associated RdRp molecule(s) [4, 30]. Viruses of the $>30$ species in this family recognized as of spring 2008 by the International Committee on Taxonomy of Viruses (ICTV) persistently infect plant or fungal hosts and are largely if not wholly ineffective at extracellular transmission. Until recently, only three genera were recognized: Partitivirus, Alphacryptovirus, and Betacryptovirus [8, 9]. Viruses infecting fungi were placed in the genus Partitivirus, and those infecting plants in the latter two genera.

Apicomplexan parasites in the genus Cryptosporidium infect a wide range of mammals, birds, and reptiles, and are a major cause of human diarrheal illness worldwide [34]. A bisegmented dsRNA virus associated with human stools containing Cryptosporidium oocysts was first reported in 1995 [7]. This virus was noted to be similar to previously described picobirnaviruses obtained from the feces of humans, other mammals, and birds, though with a smaller total genome length (3,000-3,500 vs. 4,000-4,500 bp). Subsequent nucleotide sequencing identified substantial differences between this "atypical" picobirnavirus from human feces containing Cryptosporidium oocysts and a more typical picobirnavirus from rabbits [10]. In the meantime, another laboratory group discovered a similar bisegmented dsRNA virus in propagated sporozoites from several isolates of $C$. parvum, but not (at that time) in those of other Cryptosporidium species [17]. Nucleotide sequencing and other studies from this second laboratory clarified that the $C$. parvum virus (specifically that from C. parvum KSU-1) shares a closer relationship with partitiviruses than with picobirnaviruses [15, 17]. As such, this virus would represent the only member of the family 
Partitiviridae to be yet identified from a protozoan host. Like other members of this family, the $C$. parvum virus appears to be largely if not wholly ineffective at extracellular transmission [15].

The distinguishing characteristics of the $C$. parvum virus, which we now call Cryptosporidium parvum virus 1 and abbreviate CSpV1, led us to propose a new genus in the family Partitiviridae to accommodate it. Establishment of this genus, named Cryspovirus to reflect the host genus Cryptosporidium, was considered positively by the ICTV Executive Committee at its 2008 meeting in Istanbul, Turkey, and then approved by that committee in June 2009.

\section{Taxonomic structure}

Order: Unassigned

Family: Partitiviridae

Genus: Cryspovirus

Type species: Cryptosporidium parvum virus 1

Despite being discovered a number of years ago, CSpV1 had not been formally recognized or classified by the ICTV. In association with proposing the genus Cryspovirus in the family Partitiviridae, we also proposed Cryptosporidium parvum virus 1 as the type species of this genus. High levels of partial sequence identity among CSpV1-like dsRNAs from multiple human or bovine stool samples containing $C$. parvum or $C$. hominis $[10,13,15,17,21-24$, 38] suggest that most of these dsRNAs represent different variants of the type species. On the other hand, some CSpV1-like small (CP-encoding) dsRNAs from human stool samples containing $C$. hominis, C. felis, or C. meleagridis show somewhat greater sequence divergence [21, 22] and therefore might represent additional, yet-to-beformally recognized species in the genus Cryspovirus. Criteria for distinguishing such new species would include the host species in which they naturally occur, the sizes of their genome segments and products, and the extent of amino acid sequence identity, as suggested for other members of the family Partitiviridae [8].

During review of the current proposal by the ICTV Executive Committee, a question arose as to whether the genus name Gammacryptovirus might be preferable to Cryspovirus, since the genus names Alphacryptovirus and Betacryptovirus already exist in the family Partitiviridae [8, 9]. Historically, the genera Alphacryptovirus and Betacryptovirus were created under the family Cryptoviridae of "cryptic" (asymptomatic) plant viruses. Because of similarities between members of these two genera of plant viruses and fungal viruses classified under the genus Partitivirus in the family Partitiviridae [8, 9], these three genera were later combined under the family Partitiviridae, and the family Cryptoviridae was dropped. We hence did not propose the name Gammacryptovirus for this new genus because, based on past and current precedents, it could erroneously imply that the virus being considered is a plant virus. We did entertain the genus name Cryptosporidiumvirus, but opted for Cryspovirus for brevity.

Picobirnaviruses, another group of small, bisegmented dsRNA viruses [7, 10, 35], as introduced above, were recently assigned to the family Picobirnaviridae by the ICTV. Picorbirnaviruses and partitiviruses share structural similarities in their capsids, though distinguishing features are also evident [6, 28, 29]. Other characteristics of picobirnaviruses that argue against that new family also encompassing CSpV1 include: (1) infection of vertebrate hosts, (2) probable capacity for effective extracellular transmission, (3) probable copackaging of both genome segments in the infectious virion, (4) possession of a second open reading frame (ORF) in the $\mathrm{CP}$-encoding genome segment, (5) autoproteolytic maturation of the CP [6], and (6) phylogenetically distinct $\mathrm{CP}$ and RdRp sequences.

The abbreviation CPV has been used at times for Cryptosporidium parvum virus $[12,15]$. Unfortunately, this same abbreviation has also been used for several other viruses, including cytoplasmic polyhedrosis virus, canine parvovirus, and cowpox virus. To avoid confusion, we have introduced the unique abbreviation $\mathrm{CSpV}$ for the virus(es) from $C$. parvum.

\section{Biological properties}

Like other members of the family Partitiviridae [8, 9], CSpV1 seems to be transmitted largely if not wholly by intracellular means: vertically during cell division (asexual reproduction) of Cryptosporidium host cells to produce merozoites and sporozoites, as well as during gamete fusion (sexual reproduction) preceding encystment and sporogony. Horizontal, extracellular transmission to previously uninfected Cryptosporidium cells has not been demonstrated and seems unlikely to occur with any regularity based on available observations [15]. CSpV1 is disseminated in nature within Cryptosporidium oocysts, which are produced and shed in great profusion.

Also like other members of the family Partitiviridae $[8,9], \mathrm{CSpV} 1$ is probably associated with persistent, largely avirulent infections of its hosts [15]. Some authors have thus referred to $C$. parvum as having a "viral symbiont" [18]. Although Cryptosporidium species are pathogens of humans and other vertebrates [34], there are so far no clear examples in which parasite pathogenicity is either positively or negatively modulated by infection with $\mathrm{CSpV} 1$ or another CSpV1-like virus. A recent study may spur interest in this subject, however, by showing a correlation between 
levels of CSpV1-like dsRNAs in two $C$. parvum isolates and parasite fecundity in terms of oocyst excretion from infected calves [11].

The cell biology of CSpV1 infection, including subcellular localizations of replication and assembly complexes, remains largely unexplored. Host factors that are required for, modulate, inhibit, or are affected by CSpV1 infection are also unknown.

\section{Virions and replication}

CSpV1 has isometric virions, with a buoyant density of $\sim 1.42 \mathrm{~g} / \mathrm{cm}^{3}$ on $\mathrm{CsCl}$ gradients and a diameter of $\sim 31 \mathrm{~nm}$ as visualized by negative staining and transmission electron microscopy $[15,16]$. The capsids appear single layered and thin, with a suggestion of short protrusions on their surfaces.

Genome-deduced protein sequences [17, 22] have revealed that the $\mathrm{CP}$ of $\mathrm{CSpV} 1$ is identically sized, at 319 aa or $\sim 37 \mathrm{kDa}$, to those encoded by CSpV1-like small dsRNAs from three other Cryptosporidium species. The CPs of other members of family Partitiviridae are consistently larger than this (see Table 1 for examples), suggesting that the CSpV1 CP may approach a minimal size for forming the type of 120-subunit $T=1$ (so-called " $T=2$ ") capsid recently shown to be characteristic of this family $[28,29]$. The next smallest $\mathrm{CP}$ in the family is that of Fusarium solani virus 1, at 413 aa [27]. CSpV1 CP migrates with a relative molecular weight of 37,00040,000 in denaturing gels [15] and is thus presumably full length (i.e., not proteolytically processed). An additional, 30,000-sized form of CSpV1-like CP has also been reported [18], but is likely a degradation product.
Genome-deduced protein sequences [17] have revealed that the RdRp of CSpV1 comprises 524 aa or $\sim 62 \mathrm{kDa}$, approaching the RdRp size of certain members of the family Partitiviridae such as Penicillium stoloniferum virus $\mathrm{F}$ (Table 1). In line with other members of the family Partitiviridae [4], as few as one copy of the CSpV1 RdRp is thought to be packaged per virion, probably by being noncovalently anchored inside the capsid. This virion-associated RdRp molecule is expected to mediate synthesis of the CSpV1 RNAs during transcription and replication $[14,15]$.

The genome of CSpV1 comprises two distinct molecules of dsRNA, sized near 1,700 bp (dsRNA1, large segment) and 1,400 bp (dsRNA2, small segment) in nondenaturing gels [17]. Whether these dsRNAs are $5^{\prime}$-capped at either end remains unknown, but results indicate that they are unlikely to be $3^{\prime}$-polyadenylylated $[17,22]$. Analyses performed with $\mathrm{CSpV}-1$ virions separated on $\mathrm{CsCl}$ density gradients indicate that the large and small genome segments are packaged in separate particles [15], as is true for other members of the family Partitviridae [3, 8, 9]. In some members of this family, satellite or defective RNAs can be packaged and replicated in parallel with the two essential genome segment(s), although their significance to infection is generally unknown $[8,9]$. To date, satellite or defective dsRNAs have not been reported in CSpV1.

Like those of other dsRNA viruses, the RdRp-containing particles of CSpV1 are expected to be "nanomachines" for RNA synthesis. Transcription by CSpV1 virions is asymmetric (producing only plus-strand products) and semiconservative (meaning that the parental plus strand is released while the newly synthesized plus strand is retained as part of the duplex template, that is, until the next round of transcription when it represents the parental plus strand) $[14,15]$. The primary products of transcription appear to be

Table 1 Genome segment and protein lengths for CSpV1 and representative viruses from family Partitiviridae

\begin{tabular}{|c|c|c|c|c|c|c|}
\hline \multirow[t]{2}{*}{ Virus } & \multicolumn{2}{|c|}{ GenBank accession nos. } & \multicolumn{2}{|c|}{ Genome segment lengths (bp) } & \multicolumn{2}{|c|}{ Protein lengths (aa) } \\
\hline & dsRNA1 & dsRNA2 & dsRNA1 & dsRNA2 & $\mathrm{RdRp}^{\mathrm{a}}$ & $\mathrm{CP}^{\mathrm{b}}$ \\
\hline CspV1 & U95995 & U95996 & 1,786 & 1,374 & 524 & $319^{\mathrm{c}}$ \\
\hline PsV-F & AY738336 & AY738337 & 1,677 & 1,500 & 538 & 420 \\
\hline FsV1 & D55668 & D55669 & 1,645 & 1,445 & 519 & 413 \\
\hline PsV-S & AY156521 & AY156522 & 1,754 & 1,582 & 539 & 434 \\
\hline $\mathrm{AhV}$ & L39125 & L39126 & 2,180 & 2,135 & 665 & 652 \\
\hline WCCV1 & AY705784 & AY705785 & 1,955 & 1,708 & 616 & 487 \\
\hline
\end{tabular}

CSpV1, Cryptosporidium parvum virus 1; PsV-F, Penicillium stoloniferum virus F; FsV1, Fusarium solani virus 1; PsV-S, Penicillium stoloniferum virus $\mathrm{S}$; AhV, Atkinsonella hypoxylon virus; WCCV1, White clover cryptic virus 1

${ }^{\text {a }}$ Encoded by the larger genome segment, dsRNA1, in each listed virus

b Encoded by the smaller genome segment, dsRNA2, in each listed virus

${ }^{c}$ Full-length CspV1-like CPs of identical length to CSpV1 CP have been reported from each of three other Cryptosporidium species-C. hominis, C. felis, or C. meleagridis—with GenBank accession numbers DQ193518, DQ193519, and DQ193520 
full-length copies of the genomic plus strand [14], which serve for translation by host ribosomes and also for incorporation into newly assembling virions. The CSpV1 RdRp is also presumed to mediate one round of minusstrand synthesis (replication) to generate the duplex genome molecule in newly assembled virions before switching to transcription mode. Since CSpV1 is likely to be regularly transmitted only by intracellular routes, its virions are likely to lack the machinery for cell entry.

\section{Genomic and coding properties}

Full-length nucleotide sequences have been reported for the small segment, dsRNA2, of CSpV1 [17] as well as the CSpV1-like small dsRNAs from three other Cryptosporidium species [22]. All have lengths of 1,374-1,502 bp, somewhat smaller than those of other members of family Partitiviridae (see examples in Table 1). A full-length nucleotide sequence of the large dsRNA segment, dsRNA1, has been reported only for CSpV1. It has a length of 1,786 bp, also at the small end of the range for members of family Partitiviridae (Table 1).

The genomic plus strand of each CSpV1-like dsRNA contains only one long ORF (Fig. 1). The ORF in dsRNA1 encodes RdRp, and that in dsRNA2 encodes CP. In CSpV1 dsRNA2 and the three other CSpV1-like small dsRNAs for which full-length sequences have been reported, the $5^{\prime}$ untranslated region (UTR) of the plus strand varies fairly widely in length, from $247 \mathrm{nt}$ in CSpV1 to $345-355 \mathrm{nt}$ in the others. The dsRNA2 sequence of CSpV1 [17] is missing $\sim 100 \mathrm{nt}$ at its $5^{\prime}$ terminus relative to that of the others [22], possibly due to a sequencing artifact. The $3^{\prime}$ UTR of the dsRNA2 plus strand is more consistently sized among these sequences, from 167 to $187 \mathrm{nt}$. The $5^{\prime}$ and $3^{\prime}$ UTRs of CSpV1 dsRNA1 are substantially shorter than those of its dsRNA2: 133 and $78 \mathrm{nt}$, respectively. The genomic minus strand of each appears to have little or no coding potential, consistent with its expected lack of access to host ribosomes during the viral replication cycle.

Several observations suggest that the CSpV1 CP may be translated via a noncanonical mechanism. (1) The $5^{\prime}$ UTR

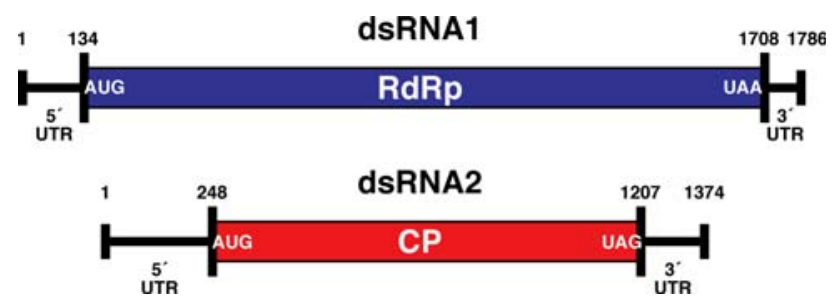

Fig. 1 Genome organization of CSpV1. The plus strand of each of the two genome segments (dsRNA1 and dsRNA2) includes one large ORF, encoding either RdRp or CP as indicated of the plus strand of CSpV1 dsRNA2 (and even more so those of the other, putative CSpV1-like viruses) is unusually long. (2) The CP start codon is in a suboptimal context for translation initiation: YYYAUGA, versus consensus AARAUGR in a set of 81 nonviral $C$. parvum genes retrieved from GenBank. (3) The translating plus strands are likely not $3^{\prime}$-polyadenylylated, and may not be $5^{\prime}$ capped. The $5^{\prime}$ UTR of the plus strand of CSpV1 dsRNA1 is shorter, and the RdRp start codon is in a more optimal context (GAAAUGA), making it less clear whether its translation mechanism may also be noncanonical.

Conserved sequences have been noted at the plus-strand $3^{\prime}$ ends of CSpV1 dsRNAs 1 and 2, including the $3^{\prime}$-terminal nonanucleotide GGGAAGCCU [14]. These sequences have been proposed to be involved in recognition by the viral RdRp during replication (minus-strand synthesis) and/or packaging into virions. Interestingly, these conserved sequences are also found in the three other CSpV1-like small dsRNAs for which full-length sequences have been reported [22], but starting 60-80 nt before the $3^{\prime}$ terminus of each. Whether this represents real variation or sequencing artifacts in one or the other study remains uncertain.

\section{Phylogenetic relationships}

Species in the family Partitiviridae recognized by the ICTV as of its 2008 meeting in Istanbul, Turkey, represent $>30$ plant and fungal viruses. Eighteen of these species have been characterized in the literature by full-length sequences of both viral genome segments, allowing phylogenetic comparisons of the, respectively, encoded proteins, RdRp and CP (Fig. 2). A clade of mostly plant viruses assigned to the genus Alphacryptovirus is evident in both phylogenetic trees, as has been previously described $[1,2]$. Genus Betacryptovirus is not represented in Fig. 2 because of the complete lack of sequences in the databases. A clade of fungal viruses assigned to the genus Partitivirus is also evident in both trees, although in this case two divergent subclades are clearly seen, as has been repeatedly noted in previous reports [1, 2, 5, 9, 25, 31-33]. Fungal viruses in the family Partitiviridae thus appear to be polyphyletic, and the genus Partitivirus may warrant future partition into two or more genera reflecting these subclades [9]. CSpV1 is well separated from each of these other clades in both phylogenetic trees (Fig. 2), supporting the conclusion that it should reside in a separate genus.

To which other group of viruses in the family Partitiviridae is CSpV1 most closely related? Although the answer is not yet definitive because of low bootstrap-support values in Fig. 2, the results to date suggest that CSpV1 is most closely related to the subclade of the genus Partitivirus containing Penicillium stoloniferum viruses $\mathrm{F}$ 


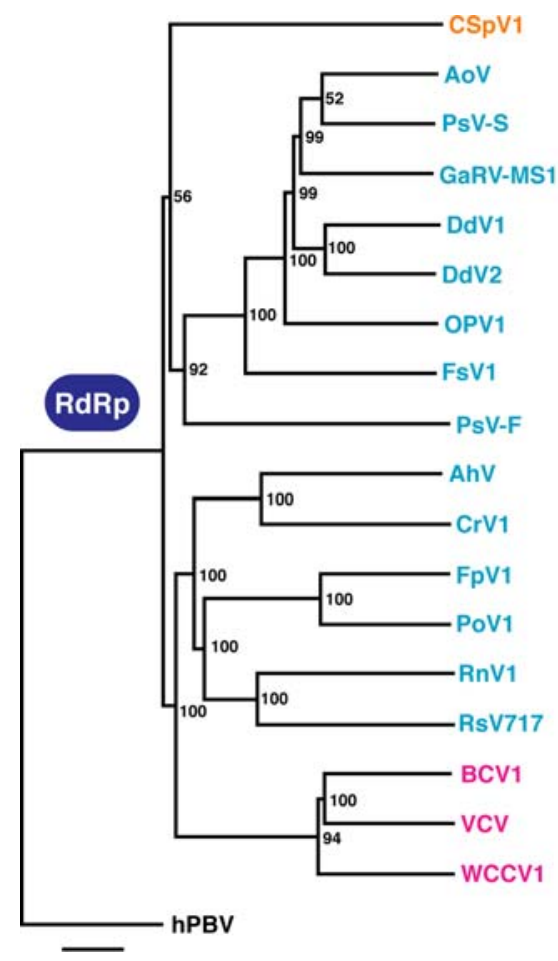

Fig. 2 Phylogenetic trees based on the complete RdRp (left) and CP (right) sequences of each analyzed virus. Sequences were multiply aligned using M-Coffee version 6.0.7 as implemented at http://www.tcoffee.org [26, 36]. Neighbor-joining trees were then generated using PAUP* (version 4.0b10) and plotted as phylograms using FigTree (version 1.1.2). Each tree was rooted by designating human picobirnavirus (hPBV) as outgroup. Respective consensus trees obtained from 2,000 bootstrap replicates showed identical topologies to the displayed phylograms, and the bootstrap percentage values are indicated at the nodes. Scale bars indicate distance in units of 0.1 substitutions per aa position. Virus names are color coded in the on-line version to reflect current genus assignments in the family Partitiviridae (see text for further discussion): genus Cryspovirus (top 1 virus, orange); genus Partitivirus (next 14 viruses, cyan); genus Alphacryptovirus (bottom 3 viruses other than hPBV, magenta). Certain of the virus abbreviations and GenBank accession numbers are defined in Table 1. Other ICTV-recognized members of family

and S. Pairwise comparisons of the $\mathrm{CP}$ and $\mathrm{RdRp}$ sequences of CSpV1 with those of partitivirus Penicillium stoloniferum virus $\mathrm{F}$ (19 and 23\% identity, respectively), partitivirus Atkinsonella hypoxylon virus (15 and 18\% identity, respectively), and alphacryptovirus white clover cryptic virus 1 (16 and 20\% identity, respectively) support this conclusion.

\section{Practical uses for host studies}

Because human infections by $C$. parvum or hominis are commonly linked to consumption of fecally oocyst-contaminated water, sensitive methods to detect the oocysts in potential sources are important. These methods include detection of CSpV1-like CPs by immunologic means such

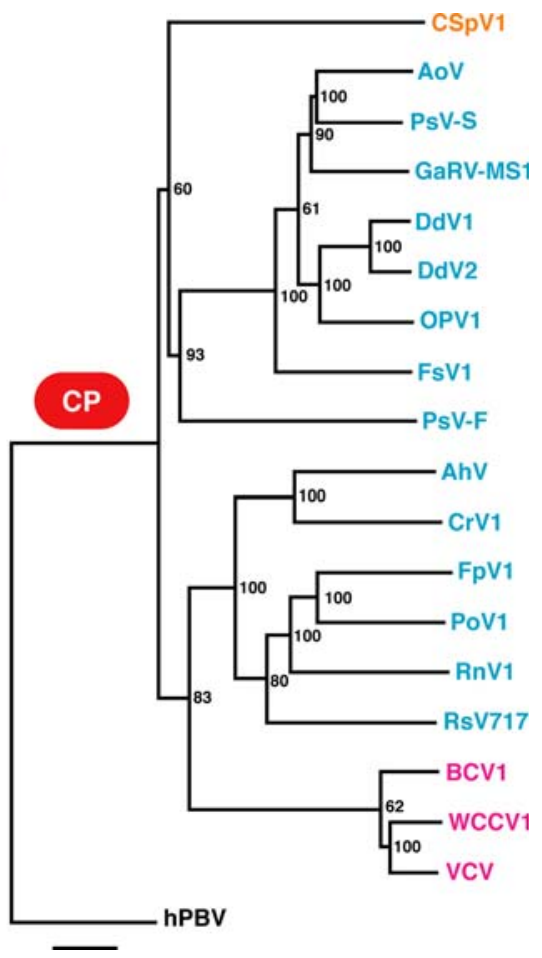

Partitiviridae included in the trees are: AoV, Aspergillus ochraceous virus (EU118277 and EU118278); PsV-S, Penicillium stoloniferm virus S (AY156521 and AY156522); GaRV-MS1, Gremmeniella abietina RNA virus MS1 (AY089993 and AY089994); DdV1, Discula destructiva virus 1 (AF316992 and AF316993); DdV2, Discula destructiva virus 2 (AY033436 and AY033437); OPV1, Ophiostoma partitivirus 1 (AM087202 and AM087203); FsV1, Fusarium solani virus 1 (D55668 and D55669); CrV1, Ceratocystis resinifera virus 1 (AY603051 and AY603052); FpV1, Fusarium poae virus 1 (AF015924 and AF047013); PoV1, Pleurotus ostreatus virus 1 (AY533036 and AY533038); RnV1, Rosellinia necatrix virus 1 (AB113347 and AB113348); RsV717, Rhizoctonia solani virus 717 (AF133290 and AF133291); BCV1, beet cryptic virus 1 (EU489061 and EU489062); and VCV, Vicia cryptic virus (AY751737 and AY751738). GenBank accession numbers for hPBV are AB186897 and $\mathrm{AB} 186898$

as dot blotting $[12,18,19]$ and detection of CSpV1-like dsRNAs by reverse transcription and polymerase chain reaction [20]. Whether the oocysts detected in contaminated sources remain viable for infection is another important question, and a correlation between loss of parasite viability and loss of detectable CSpV1-like dsRNAs may be useful in this regard [16].

The association with contaminated water means that human infections by $C$. parvum or hominis can occur in epidemic-like outbreaks [34]. Detection is not, therefore, the only relevant issue, but also epidemiological tracking to link the specific parasite isolates in patients to those in potential sources. Sequences of CSpV1-like genome fragments amplified by reverse transcription and polymerase chain reaction have proven useful in this regard [21, 23, 24, 38]. CSpV1-like sequences have also been useful in 
ongoing efforts to clarify the diversity and taxonomy of Cryptosporidium species, including the partitioning of C. hominis from C. parvum [13, 37].

Both $C$. parvum and $C$. hominis are also commonly associated with direct person-to-person transmission, and C. parvum with direct animal-to-animal transmission (calf to calf, cow to calf, etc.), again through oocyst-contaminated fecal material. For example, in cases of chronic cryptosporidiosis accompanying AIDS and other immunosuppressive illnesses [34], oocyst shedding over long periods raises particular concerns for transmission. Methods for detection and epidemiologic tracking of C. parvum and hominis are clearly important in such cases of direct transmission as well.

\section{References}

1. Blawid R, Stephan D, Maiss E (2007) Molecular characterization and detection of Vicia cryptic virus in different Vicia faba cultivars. Arch Virol 152:1477-1488

2. Boccardo G, Candresse $T$ (2005) Complete sequence of the RNA1 of an isolate of White clover cryptic virus 1, type species of the genus Alphacryptovirus. Arch Virol 150:399-402

3. Buck KW, Kempson-Jones GF (1973) Biophysical properties of Penicillium stoloniferum virus S. J Gen Virol 18:223-235

4. Buck KW, Kempson-Jones GF (1974) Capsid polypeptides of two viruses isolated from Penicillium stoloniferum. J Gen Virol 22:441-445

5. Crawford LJ, Osman TA, Booy FP, Coutts RH, Brasier CM, Buck KW (2006) Molecular characterization of a partitivirus from Ophiostoma himal-ulmi. Virus Genes 33:33-39

6. Duquerroy S, Da Costa B, Henry C, Vigouroux A, Libersou S, Lepault J, Navaza J, Delmas B, Rey FA (2009) The picobirnavirus crystal structure provides functional insights into virion assembly and cell entry. EMBO 28:1655-1665

7. Gallimore CI, Green J, Casemore DP, Brown DWG (1995) Detection of a picobirnavirus associated with Cryptosporidium positive stools from humans. Arch Virol 140:1275-1278

8. Ghabrial SA, Buck KW, Hillman BI, Milne RG (2005) Partitiviridae. In: Fauquet CM, Mayo MA, Maniloff J, Desselberger U, Ball LA (eds) Virus taxonomy: eighth report of the International Committee on Taxonomy of Viruses. Elsevier Academic Press, San Diego, pp 581-590

9. Ghabrial SA, Ochoa WF, Baker TS, Nibert ML (2008) Partitiviruses. In: Mahy BWJ, Van Regenmortel MHV (eds) Encyclopedia of virology, vol 4, 3rd edn. Elsevier Academic Press, San Diego, pp 68-75

10. Green J, Gallimore CI, Clewley JP, Brown DWG (1999) Genomic characterisation of the large segment of a rabbit picobirnavirus and comparison with the atypical picobirnavirus of Cryptosporidium parvum. Arch Virol 144:2457-2465

11. Jenkins MC, Higgins J, Abrahante JE, Kniel KE, O'Brien C, Trout J, Lancto CA, Abrahamsen MS, Fayer R (2008) Fecundity of Cryptosporidium parvum is correlated with intracellular levels of the viral symbiont CPV. Int J Parasitol 38:1051-1055

12. Jenkins MC, O'Brien CN, Trout JM (2008) Detection of Cryptosporidium parvum oocysts by dot-blotting using monoclonal antibodies to Cryptosporidium parvum virus $40-\mathrm{kDa}$ capsid protein. J Parasitol 94:94-98
13. Khramtsov NV, Chung PA, Dykstra CC, Griffiths JK, Morgan UM, Arrowood MJ, Upton SJ (2000) Presence of double-stranded RNAs in human and calf isolates of Cryptosporidium parvum. J Parasitol 86:275-282

14. Khramtsov NV, Upton SJ (1998) High-temperature inducible cell-free transcription and replication of double-stranded RNAs within the parasitic protozoan Cryptosporidium parvum. Virology 245:331-337

15. Khramtsov NV, Upton SJ (2000) Association of RNA polymerase complexes of the parasitic protozoan Cryptosporidium parvum with virus-like particles: heterogeneous system. J Virol 74:5788-5795

16. Khramtsov NV, Upton SJ (2003) dsRNAs of Cryptosporidium parvum. J Parasitol 89:S165-S168

17. Khramtsov NV, Woods KM, Nesterenko MV, Dykstra CC, Upton SJ (1997) Virus-like, double-stranded RNAs in the parasitic protozoan Cryptosporidium parvum. Mol Microbiol 26:289-300

18. Kniel KE, Higgins JA, Trout JM, Fayer R, Jenkins MC (2004) Characterization and potential use of a Cryptosporidium parvum virus (CPV) antigen for detecting $C$. parvum oocysts. J Microbiol Methods 58:189-195

19. Kniel KE, Jenkins MC (2005) Detection of Cryptosporidium parvum oocysts on fresh vegetables and herbs using antibodies specific for a Cryptosporidium parvum viral antigen. J Food Prot 68:1093-1096

20. Kozwich D, Johansen KA, Landau K, Roehl CA, Woronoff S, Roehl PA (2000) Development of a novel, rapid integrated Cryptosporidium parvum detection assay. Appl Environ Microbiol 66:2711-2717

21. Leoni F, Gallimore CI, Green J, McLauchlin J (2003) Molecular epidemiological analysis of Cryptosporidium isolates from humans and animals by using a heteroduplex mobility assay and nucleic acid sequencing based on a small double-stranded RNA element. J Clin Microbiol 41:981-992

22. Leoni F, Gallimore CI, Green J, McLauchlin J (2006) Characterisation of small double stranded RNA molecule in Cryptosporidium hominis, Cryptosporidium felis and Cryptosporidium meleagridis. Parasitol Int 55:299-306

23. Leoni F, Gómez-Couso H, Ares-Mazás ME, McLauchlin J (2007) Multilocus genetic analysis of Cryptosporidium in naturally contaminated bivalve molluscs. J Appl Microbiol 103:2430-2437

24. Leoni F, Mallon ME, Smith HV, Tait A, McLauchlin J (2007) Multilocus analysis of Cryptosporidium hominis and Cryptosporidium parvum isolates from sporadic and outbreak-related human cases and $C$. parvum isolates from sporadic livestock cases in the United Kingdom. J Clin Microbiol 45:3286-3294

25. Lim WS, Jeong JH, Jeong RD, Yoo YB, Yie SW, Kim KH (2005) Complete nucleotide sequence and genome organization of a dsRNA partitivirus infecting Pleurotus ostreatus. Virus Res 108:111-119

26. Moretti S, Armougom F, Wallace IM, Higgins DG, Jongeneel CV, Notredame C (2007) The M-coffee web server: a metamethod for computing multiple sequence alignments by combining alternative alignment methods. Nucleic Acids Res 35:W645-W648

27. Nogawa M, Kageyama T, Nakatani A, Taguchi G, Shimosaka M, Okazaki M (1996) Cloning and characterization of mycovirus double-stranded RNA from the plant pathogenic fungus, Fusarium solani f. sp. robiniae. Biosci Biotechnol Biochem 60:784788

28. Ochoa WF, Havens WM, Sinkovits RS, Nibert ML, Ghabrial SA, Baker TS (2008) Partitivirus structure reveals a 120-subunit, helix-rich capsid with distinctive surface arches formed by quasisymmetric coat-protein dimers. Structure 16:776-786

29. Pan J, Dong L, Lin L, Ochoa WF, Sinkovits RS, Havens WM, Nibert ML, Baker TS, Ghabrial SA, Tao YJ (2009) Atomic 
structure reveals the unique capsid organization of a dsRNA virus. Proc Natl Acad Sci USA 106:4225-4230

30. Ratti G, Buck KW (1978) Semi-conservative transcription in particles of a double-stranded RNA mycovirus. Nucleic Acids Res 5:3843-3854

31. Rong R, Rao S, Scott SW, Carner GR, Tainter FH (2002) Complete sequence of the genome of two dsRNA viruses from Discula destructiva. Virus Res 90:217-224

32. Sasaki A, Miyanishi M, Ozaki K, Onoue M, Yoshida K (2005) Molecular characterization of a partitivirus from the plant pathogenic ascomycete Rosellinia necatrix. Arch Virol 150:1069-1083

33. Tuomivirta TT, Hantula J (2003) Two unrelated double-stranded RNA molecule patterns in Gremmeniella abietina type A code for putative viruses of the families Totiviridae and Partitiviridae. Arch Virol 148:2293-2305
34. Tzipori S, Widmer G (2008) A hundred-year retrospective on cryptosporidiosis. Trends Parasitol 24:184-189

35. Wakuda M, Pongsuwanna Y, Taniguchi K (2005) Complete nucleotide sequences of two RNA segments of human picobirnavirus. J Virol Methods 126:165-169

36. Wallace IM, O'Sullivan O, Higgins DG, Notredame C (2006) M-Coffee: combining multiple sequence alignment methods with T-coffee. Nucleic Acids Res 34:1692-1699

37. Xiao L, Fayer R, Ryan U, Upton SJ (2004) Cryptosporidium taxonomy: recent advances and implications for public health. Clin Microbiol Rev 17:72-97

38. Xiao L, Limor J, Bern C, Lal AA (2001) Tracking Cryptosporidium parvum by sequence analysis of small double-stranded RNA. Emerg Infect Dis 7:141-145 